\title{
Disjoint unions of dimensional dual hyperovals
}

\author{
Satoshi Yoshiara
}

\begin{abstract}
The notion of sub-objects and their disjoint union is introduced for a dimensional dual arc (Section 1.2). This naturally motivates a problem to decompose a dimensional dual hyperoval (DHO for short) into the disjoint union of some subdual arcs, including a subDHO (Section 1.3), because such an expression is useful to calculate its universal cover, as suggested by an elementary observation about its ambient space (Proposition 2.1). Under mild restrictions, a criterion is obtained for a DHO $\mathcal{B}_{1}$ of rank $n$ over two element field to be extended to a DHO $\mathcal{A}$ of rank $n+1$ so that $\mathcal{A}$ is a disjoint union of $\mathcal{B}_{1}$ and some subDHO $\mathcal{B}_{2}$ of rank $n$ (Theorem 1.3(i)). Under the choice of a complement to $\mathcal{B}_{1}$, such $\mathcal{A}$ as well as $\mathcal{B}_{2}$ are uniquely determined by $\mathcal{B}_{1}$, if they exist (Theorem 1.3(ii)). Several known families of DHOs are examined whether they can be extended to DHOs in the above form, but no example is found unless they are bilinear. If a subDHO $\mathcal{B}_{1}$ is bilinear over a specified complement, the criterion is satisfied, and thus there exists a unique pair $\left(\mathcal{A}, \mathcal{B}_{2}\right)$ of DHOs satisfying the above conditions (Corollary 1.4). This makes clear the meaning of the construction, called "extension" in [1, Section 5] for bilinear DHOs.
\end{abstract}

Keywords: dimensional dual arc (DA), dimensional dual hyperoval (DHO), subDA, disjoint union of subDA, cover, bilinear DHO, o-polynomial.

MSC 2010: 05B25, 51A45, 51E20

\section{Introduction}

\subsection{Dimensional dual arcs (DA) and hyperovals (DHO)}

For a natural number $n$ with $n \geq 2$, a dimensional dual $\operatorname{arc}$ (DA, for short) of rank $n$ over a finite field $\mathbb{F}_{q}$ is a nonempty collection $\mathcal{A}$ of subspaces of a vector 

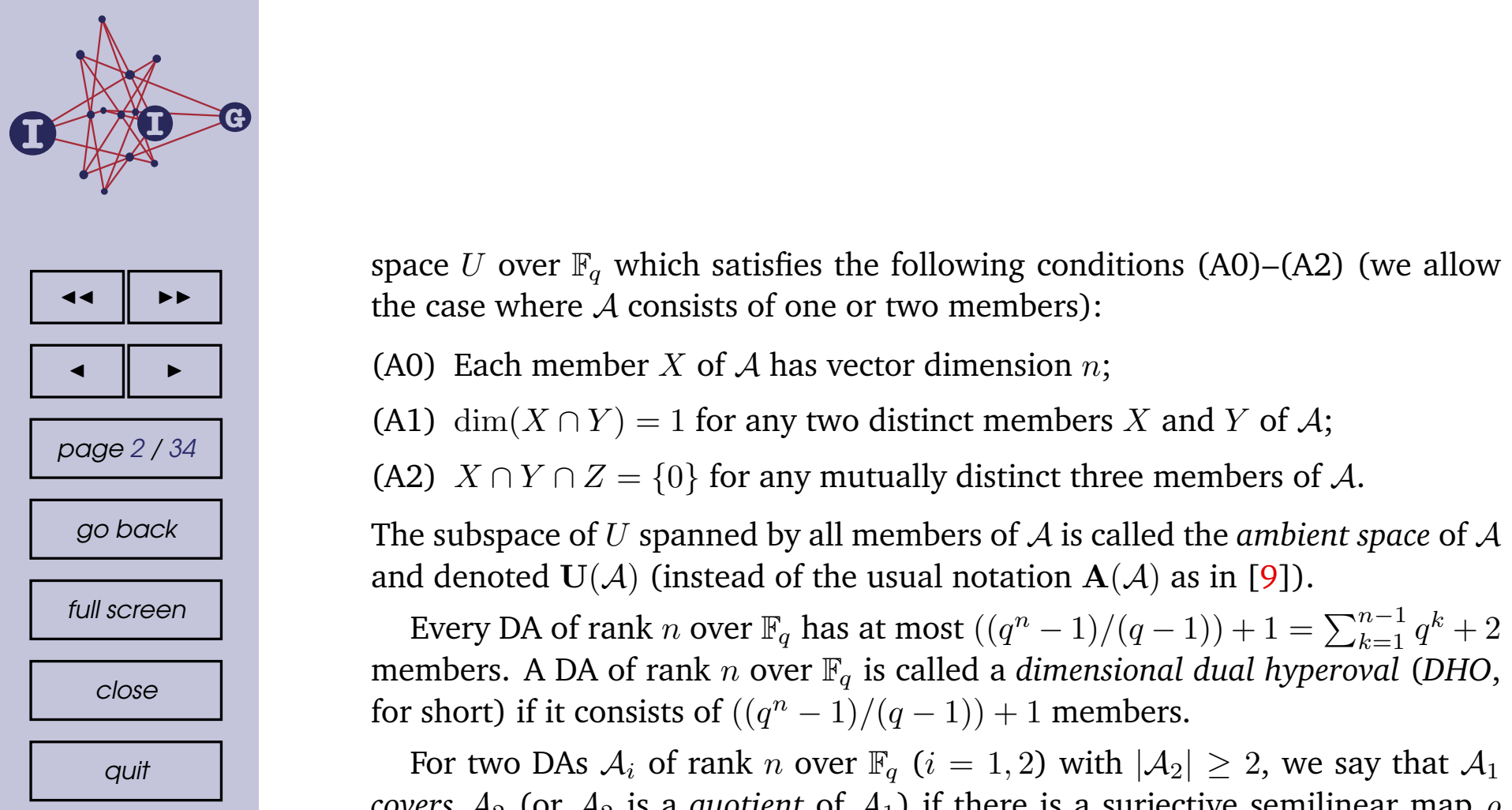
the case where $\mathcal{A}$ consists of one or two members):

(A0) Each member $X$ of $\mathcal{A}$ has vector dimension $n$;

(A1) $\operatorname{dim}(X \cap Y)=1$ for any two distinct members $X$ and $Y$ of $\mathcal{A}$;

(A2) $X \cap Y \cap Z=\{0\}$ for any mutually distinct three members of $\mathcal{A}$.

The subspace of $U$ spanned by all members of $\mathcal{A}$ is called the ambient space of $\mathcal{A}$ and denoted $\mathbf{U}(\mathcal{A})$ (instead of the usual notation $\mathbf{A}(\mathcal{A})$ as in [9]).

Every DA of rank $n$ over $\mathbb{F}_{q}$ has at most $\left(\left(q^{n}-1\right) /(q-1)\right)+1=\sum_{k=1}^{n-1} q^{k}+2$ members. A DA of rank $n$ over $\mathbb{F}_{q}$ is called a dimensional dual hyperoval (DHO, for short) if it consists of $\left(\left(q^{n}-1\right) /(q-1)\right)+1$ members.

For two DAs $\mathcal{A}_{i}$ of rank $n$ over $\mathbb{F}_{q}(i=1,2)$ with $\left|\mathcal{A}_{2}\right| \geq 2$, we say that $\mathcal{A}_{1}$ covers $\mathcal{A}_{2}$ (or $\mathcal{A}_{2}$ is a quotient of $\mathcal{A}_{1}$ ) if there is a surjective semilinear map $\rho$ from $\mathbf{U}\left(\mathcal{A}_{1}\right)$ to $\mathbf{U}\left(\mathcal{A}_{2}\right)$ (thus if $q=2, \rho$ is just an $\mathbb{F}_{2}$-linear surjection) which maps $\mathcal{A}_{1}$ surjectively onto $\mathcal{A}_{2}$ and sends each member of $\mathcal{A}_{1}$ isomorphically to a member of $\mathcal{A}_{2}$. (In particular, $\rho$ induces a bijective correspondence of the members of $\mathcal{A}_{1}$ with the members of $\mathcal{A}_{2}$.) When $\operatorname{dim}\left(\mathbf{U}\left(\mathcal{A}_{1}\right)\right)=\operatorname{dim}\left(\mathbf{U}\left(\mathcal{A}_{2}\right)\right)$, we say that $\mathcal{A}_{1}$ is isomorphic to $\mathcal{A}_{2}$ if $\mathcal{A}_{1}$ covers $\mathcal{A}_{2}$. When $\mathcal{A}_{1}=\mathcal{A}_{2}=\mathcal{A}$, a map $\rho$ on $\mathbf{U}(\mathcal{A})$ with this property is called an automorphism of $\mathcal{A}$. The set $\operatorname{Aut}(\mathcal{A})$ of all automorphisms of $\mathcal{A}$ forms a group with respect to the composition of maps, namely, $\operatorname{Aut}(\mathcal{A})$ is the group of all bijective semilinear transformations on $\mathbf{U}(\mathcal{A})$ which preserve $\mathcal{A}$. A DA $\mathcal{A}$ of rank $n$ over $\mathbb{F}_{q}$ is called simply connected if any cover of $\mathcal{A}$ coincides with $\mathcal{A}$.

The notion of covers and quotients among DAs (specifically DHOs) naturally arises as an analogue to that of the universal covers and quotients of the associated incidence geometries, and has been used as one of the main tools to investigate DAs.

\subsection{SubDAs and their disjoint unions}

On the other hand, the notion of subDAs and their (disjoint) unions attracted less attention so far, although it naturally arises in the following manner.

Let $\mathcal{A}$ be a DHO of rank $n$ over $\mathbb{F}_{q}$ with $n \geq 3$. Fix a positive integer $n^{\prime}$ satisfying $2 \leq n^{\prime}<n$. From each member $A$ of $\mathcal{A}$, we pick a subspace $B$ of dimension $n^{\prime}$ and consider their collection $\mathcal{B}$. Needless to say, there are several choices for $B$ inside each $A$, and accordingly, many possibilities for $\mathcal{B}$. However, any two distinct members of $\mathcal{B}$ do not contain a 2 -dimensional subspace in common, because their intersection is contained in two distinct members of a DA $\mathcal{A}$. Furthermore, three mutually distinct members of $\mathcal{B}$ intersect at the zero space. 



We say that a DHO $\mathcal{S}$ over $\mathbb{F}_{2}$ is bilinear over $Y$, if it splits over a subspace $Y$ and there is an ordering on the vector space $X$ parametrizing the members of $\mathcal{S}$ such that the linear system $\mathcal{L}_{X}(\mathcal{S}, Y)=\{L(t) \mid t \in X\}$ for $\mathcal{S}$ over $Y$ satisfies the following equation:

$$
L(s+t)=L(s)+L(t) \text { for any } s, t \in X .
$$

go back

full screen

close

quit

ACADEMIA

PRESS

NW

$\widehat{\underline{\text { IIIIII }}}$

UNIVERSITEIT

GENT

Notice that this notion does depend on the particular choice of a complement $Y$ to $\mathcal{S}$. (See examples in Section 3.3.) On the other hand, a DHO $\mathcal{S}$ is called bilinear if there exists a complement over which $\mathcal{S}$ is bilinear. Needless to say, this notion depends only on $\mathcal{S}$.

\subsection{Main results}

Now we state our main result, which gives an almost complete solution to the problem in Section 1.3 for $q=2=m$. This gives a criterion for a DHO $\mathcal{B}_{1}$ of rank $n$ over $\mathbb{F}_{2}$ to be a subDHO of a DHO $\mathcal{A}$ of rank $n+1$ over $\mathbb{F}_{2}$ with $\mathcal{A}=\mathcal{B}_{1} \sqcup \mathcal{B}_{2}$ for some $\mathrm{DHO} \mathcal{B}_{2}$ of rank $n$.

Theorem 1.3. Let $\mathcal{B}_{1}$ be a DHO of rank $n(n \geq 3)$ over $\mathbb{F}_{2}$ whose members are subspaces of a vector space $U$ with $\operatorname{dim}(U) \geq \operatorname{dim} \mathbf{U}\left(\mathcal{B}_{1}\right)+n+1$. Fix a member $B_{1}$ of $\mathcal{B}_{1}$ and take a subspace $A_{2}$ of $U$ of dimension $n+1$ with $A_{2} \cap \mathbf{U}\left(\mathcal{B}_{1}\right)=\{0\}$ as well as a hyperplane $B_{2}$ of $A_{2}$. We assume that $\mathcal{B}_{1}$ is of split type. Then the following hold.

(i) There exists a pair $\left(\mathcal{B}_{2}, \mathcal{A}\right)$ of DHOs $\mathcal{B}_{2}$ and $\mathcal{A}$ of rank $n$ and $n+1$, respectively, over $\mathbb{F}_{2}$ which satisfies the following conditions

$$
\mathbf{U}(\mathcal{A}) \subseteq U, B_{2} \in \mathcal{B}_{2}, A_{2} \in \mathcal{A},
$$

$\mathcal{B}_{1}$ and $\mathcal{B}_{2}$ are subDHOs of $\mathcal{A}$ with $\mathcal{A}=\mathcal{B}_{1} \sqcup \mathcal{B}_{2}$,

if and only if the members of $\mathcal{B}_{1}$ are parametrized by $B_{2}$ (so that each member of $\mathcal{B}_{1}$ is expressed as $B_{1}(c)$ for $\left.\left.c \in B_{2}\right\}\right)$ such that $B_{1}(0)=B_{1}$ and

$$
b_{1}\left\{d+x_{1}+x_{2}, x_{1}+x_{2}\right\}=b_{1}\left\{d+x_{1}, x_{1}\right\}+b_{1}\left\{d+x_{2}, x_{2}\right\}+b_{1}\{d, 0\}
$$

for all $d \in B_{2}^{\times}=B_{2} \backslash\{0\}$ and all $x_{1}, x_{2} \in B_{2}$, where $b_{1}\{c, d\}$ denotes the unique nonzero vector of $B_{1}(c) \cap B_{1}(d)$ for distinct $c, d \in B_{2}$.

(ii) Fix a complement $Y_{1}$ to $\mathcal{B}_{1}$ and a vector $a_{12}$ of $A_{2} \backslash B_{2}$. If there is a parametrization of the members of $\mathcal{B}_{1}$ by $B_{2}$ such that $B_{1}(0)=B_{1}$ and equation (3) holds for all $d \in B_{2}^{\times}$and all $x_{1}, x_{2} \in B_{2}$, the pair $\left(\mathcal{B}_{2}, \mathcal{A}\right)$ in Claim (i) with $A_{1}:=\left\langle a_{12}, B_{1}\right\rangle \in \mathcal{A}$ is uniquely determined by $\mathcal{B}_{1}$ as follows: $\mathcal{A}=\left\{A_{1}(c), A_{2}(d) \mid c, d \in B_{2}\right\}$ and $\mathcal{B}_{2}=\left\{B_{2}(c) \mid c \in B_{2}\right\}$ with 

$\mathcal{L}_{B_{2} \times \mathbb{F}_{2}}\left(\mathcal{A}, Y_{\rho}\right)$ for $\mathcal{A}$ over $Y_{\rho}$ consists of linear maps $L(c, \delta)$ given below $(c \in$ $B_{2}, \delta \in \mathbb{F}_{2}$ ): for $\varepsilon \in \mathbb{F}_{2}$ and $y \in B_{1}$,

$$
\begin{aligned}
& \left(\varepsilon a_{12}+y\right) L(c, 0):=\varepsilon c+\varepsilon c \rho+(y+\varepsilon c \rho) L_{1}(c), \\
& \left(\varepsilon a_{12}+y\right) L(c, 1):=\left(\varepsilon \kappa_{c}+y\right) \rho^{-1}+\left(\varepsilon \kappa_{c}+y\right)+\kappa_{c} L_{1}\left(\left(\varepsilon \kappa_{c}+y\right) \rho^{-1}\right) .
\end{aligned}
$$

(iii) If $\mathcal{A}$ is bilinear over $Y_{\rho}$, then we have

$$
\text { (c) } \rho L_{1}(d)=(d) \rho L_{1}(c) \text { for any } c, d \in B_{2} .
$$

full screen

close

If this equation holds, then we have $L\left(c_{1}, 0\right)+L\left(c_{2}, 0\right)=L\left(c_{1}+c_{2}, 0\right)$, $L\left(c_{1}, 1\right)+L\left(c_{2}, 1\right)=L\left(\left(\kappa_{c_{1}}+\kappa_{c_{2}}\right) \rho^{-1}, 0\right)$ for any $c_{1}, c_{2} \in B_{2}$, and the two subDHOs $\mathcal{B}_{1}$ and $\mathcal{B}_{2}$ are isomorphic.

So far, the author did not find any example of DHOs which satisfies the criterion given in Theorem 1.3(i) but is not bilinear over any complement. It is an interesting problem to prove or disprove the following statement: if the criterion given in Theorem 1.3(i) is satisfies for a DHO $\mathcal{B}_{1}$ of split type then $\mathcal{B}$ is bilinear over some complement.

\subsection{Some remarks}

We conclude this section with several remarks about Theorem 1.3 and Corollary 1.4 .

First, observe that the criterion given in Theorem 1.3(i) (the requirement that equation (3) holds for all $d, x_{1}, x_{2} \in B_{2}$ with $d \neq 0$ ) is a property for the given DHO $\mathcal{B}_{1}$, independent of the choice of the particular complement $Y_{1}$ to $\mathcal{B}_{1}$, but depending on the choice of a particular member $B_{1}$ (parametrized by $0 \in B_{2}$ ) and the ordering of $B_{2}^{\times}$.

On the other hand, the DHO $\mathcal{A}$ in Theorem 1.3(ii) is uniquely determined by the DHO $\mathcal{B}_{1}$, if we fix the following four subspaces of $U$ and a vector $a_{12}$ : a member $B_{1}$ of $\mathcal{B}_{1}$, a complement $Y_{1}$ to $\mathcal{B}_{1}$, a subspace $A_{2}$ of dimension $n+1$ with $A_{2} \cap \mathbf{U}\left(\mathcal{B}_{1}\right)=\{0\}$, a hyperplane $B_{2}$ of $A_{2}$ and $a_{12} \in A_{2} \backslash B_{2}$.

Thus we can denote $\mathcal{A}=\mathcal{A}\left(B_{1}, Y_{1} ; A_{2}, B_{2}, A_{12}\right)$. It follows from the above remark that if $\mathcal{A}\left(B_{1}, Y_{1} ; A_{2}, B_{2}, a_{12}\right)$ exists then $\mathcal{A}\left(B_{1}, Y_{1}^{\prime} ; A_{2}, B_{2}, a_{12}\right)$ exists for any complement $Y_{1}^{\prime}$ to $\mathcal{B}_{1}$.

Another choice $\left(A_{2}^{\prime}, B_{2}^{\prime}, a_{12}^{\prime}\right)$ for subspaces $U$ satisfying $\operatorname{dim}\left(A_{2}^{\prime}\right)=n+1$, $A_{2}^{\prime} \cap \mathbf{U}\left(\mathcal{B}_{1}\right)=\{0\}, \operatorname{dim}\left(B_{2}^{\prime}\right)=n, B_{2}^{\prime} \subset A_{2}^{\prime}$ and a vector $a_{12}^{\prime} \in A_{2}^{\prime} \backslash B_{2}^{\prime}$ gives a DHO $\mathcal{A}^{\prime}=\mathcal{A}\left(B_{1}, Y_{1} ; A_{2}^{\prime}, B_{2}^{\prime}, a_{12}^{\prime}\right)$, which is shown to be isomorphic to $\mathcal{A}$. More generally, if there is an automorphism $\rho_{1}$ of $\mathcal{B}_{1}$ with $B_{1}^{\prime}:=\left(B_{1}\right) \rho_{1}$, then $Y_{1}^{\prime}:=\left(Y_{1}\right) \rho_{1}$ is another complement to $\mathcal{B}_{1}$ and $\mathcal{A}\left(Y_{1}^{\prime} ; B_{1}^{\prime}, A_{2}^{\prime}, B_{2}^{\prime}, a_{12}^{\prime}\right)$ is isomorphic to the original $\mathcal{A}$, because for a linear bijection $\rho_{2}$ from $A_{2}$ onto $A_{2}^{\prime}$ with 




\subsection{Proof of Proposition $\mathbf{1 . 2}$}

(1) As $\mathcal{A}$ is a disjoint union of $\mathcal{A}\left(\mathcal{B}_{j}\right)$ for $j=1, \ldots, m$, it follows from the assumption that

$$
\frac{q^{n+1}-1}{q-1}+1=|\mathcal{A}|=\sum_{j=1}^{m}\left|\mathcal{A}\left(\mathcal{B}_{j}\right)\right|=\sum_{j=1}^{m}\left|\mathcal{B}_{j}\right|=\sum_{j=1}^{m}\left(\frac{q^{n}-1}{q-1}+1\right) .
$$

Thus $\sum_{k=1}^{n} q^{k}+2=m\left(\left(\sum_{l=1}^{n-1} q^{l}\right)+2\right)$, from which we have

$$
q^{n}=(m-1)\left(\left(\sum_{l=1}^{n-1} q^{l}\right)+2\right) .
$$

This implies that $\left(q^{n-1}+\cdots+q\right)+2$ should divide $q^{n}$. As $q=p^{e}$ for a prime $p$ and an integer $e \geq 1$, we have $\left(q^{n-1}+\cdots+q\right)+2=p^{f}$ for some $e<f \leq e n$. If $p$ is an odd prime, this equation implies that 2 is congruent to 0 modulo $p$, which is impossible. If $p=2$ and $e \geq 2$, this equation implies that 2 is congruent to 0 modulo 4 , which is impossible. Thus we should have $q=p=2$. Then $q^{n}=2^{n}$ and $\left(\left(q^{n}-1\right) /(q-1)\right)+1=2^{n}$, and hence $m=2$ by equation (6).

(2) By Proposition 2.1(2), the ambient space $\mathbf{U}(\mathcal{A})$ is spanned by $\mathbf{U}\left(\mathcal{B}_{1}\right), \mathbf{U}\left(\mathcal{B}_{2}\right)$, and a single 1-dimensional subspace $A_{1} \cap A_{2}$ for members $A_{j}$ of $\mathcal{A}\left(\mathcal{B}_{j}\right)$ $(j=1,2)$. Let $B_{2}$ be the unique member of $\mathcal{B}_{2}$ contained in $A_{2}$. Take any member $B^{\prime}$ of $\mathcal{B}_{2} \backslash\left\{B_{2}\right\}$. In order to establish the claim, it suffices to show that $B^{\prime}$ is contained in the space spanned by the members of $\mathcal{B}_{1}$ and $A_{2}$. Let $A^{\prime}$ be unique member of $\mathcal{A}$ containing $B^{\prime}$. Since $\mathcal{B}_{2}$ is a DHO, any member of $\mathcal{A}$ containing a 1-dimensional subspace of $B^{\prime}$ lies in $\mathcal{A}\left(\mathcal{B}_{2}\right)$. Thus for every $A$ of $\mathcal{A}\left(\mathcal{B}_{1}\right)$, the intersection $A \cap A^{\prime}$ does not lie in $B^{\prime}$. As $\left|\mathcal{A}\left(\mathcal{B}_{1}\right)\right|=2^{n}$, the intersections $A \cap A^{\prime}$ for $A \in \mathcal{A}\left(\mathcal{B}_{1}\right)$ exhaust all 1-dimensional subspaces of $A^{\prime}$ outside a hyperplane $B^{\prime}$. In particular, they span $A^{\prime}$. Thus $A^{\prime}$, and hence $B^{\prime}$ lies in $\mathbf{U}\left(\mathcal{A}\left(\mathcal{B}_{1}\right)\right)$. The latter is contained in $\mathbf{U}\left(\mathcal{B}_{1}\right)$ and $A_{2}$ by Proposition 2.1(1). This established the claim.

(3) Let $\tilde{\mathcal{A}}$ be a DHO which covers $\mathcal{A}$. Then there exists a linear surjection $\rho$ from $\mathbf{U}(\tilde{\mathcal{A}})$ onto $\mathbf{U}(\mathcal{A})$ which induces an isomorphism from each member $\tilde{A}$ of $\tilde{\mathcal{A}}$ to a member of $\mathcal{A}$. Notice that the inverse images of members of $\mathcal{B}_{j}$ form a subDHO, denoted $\tilde{\mathcal{B}}_{j}$, in $\tilde{\mathcal{A}}$ for each $j=1,2$, and that $\tilde{\mathcal{A}}=\tilde{\mathcal{B}}_{1} \sqcup \tilde{\mathcal{B}}_{2}$. Thus we may apply claim (2) of Proposition 1.2 to conclude that $\mathbf{U}(\tilde{\mathcal{A}})$ is spanned by the members of $\tilde{\mathcal{B}}_{1}$ and the inverse image $\rho^{-1}\left(A_{2}\right)$ of $A_{2}$, which is isomorphic to $A_{2}$. As $\mathcal{B}_{1}$ is simply connected, the ambient space spanned by the members of $\tilde{\mathcal{B}}_{1}$ is identical to that of $\mathcal{B}_{1}$. Thus we have

$$
\operatorname{dim}(\mathbf{U}(\tilde{\mathcal{A}})) \leq \operatorname{dim}\left(\mathbf{U}\left(\mathcal{B}_{1}\right)\right)+\operatorname{dim}\left(A_{2}\right)=\operatorname{dim}\left(\mathbf{U}\left(\mathcal{B}_{1}\right)\right)+n+1 .
$$


As the last number is equal to the dimension of $\mathbf{U}(\mathcal{A})$ by the assumption, we conclude that $\mathbf{U}(\tilde{\mathcal{A}})=\mathbf{U}(\mathcal{A})$. Thus $\mathcal{A}$ is simply connected.

\subsection{Proof of Theorem $\mathbf{1 . 3}$}

We recall the setting in Theorem 1.3. Let $n$ be a natural number with $n \geq 3$, and let $\mathcal{B}_{1}$ be an arbitrary DHO over $\mathbb{F}_{2}$ of rank $n$ whose members are subspaces of a vector space $U$ over $\mathbb{F}_{2}$ of dimension at least $\operatorname{dim}\left(\mathbf{U}\left(\mathcal{B}_{1}\right)\right)+n+1$. Take a subspace $A_{2}$ of $U$ of dimension $n+1$ with

(u) $A_{2} \cap \mathbf{U}\left(\mathcal{B}_{1}\right)=\{0\}$.

Fix a hyperplane $B_{2}$ of $A_{2}$. We also assume that

(s1) $\mathcal{B}_{1}$ splits over a subspace $Y_{1}$ of $\mathbf{U}\left(\mathcal{B}_{1}\right)$ of codimension $n$.

We fix a member $B_{1}$ of $\mathcal{B}_{1}$. By assumption (s1), we have $\mathbf{U}\left(\mathcal{B}_{1}\right)=B_{1} \oplus Y_{1}$. As $B_{2}$ is of dimension $n$ over $\mathbb{F}_{2}$, we may adopt $B_{2}$ as a set to parametrize the members of a DHO $\mathcal{B}_{1}$ of rank $n$ over $\mathbb{F}_{2}$ and so the linear system of a DHO $\mathcal{B}_{1}$ over $Y_{1}$ as well. (See Section 1.5.) We denote this system by $\mathcal{L}_{B_{2}}\left(\mathcal{B}_{1}, Y_{1}\right):=$ $\left\{L_{1}(c) \mid c \in B_{2}\right\}$. We take any parametrization of the members of $\mathcal{B}_{1}$ by $B_{2}$ with $B_{1}(0)=B_{1}$ and $L_{1}(0)=0$, and fix it in the sequel.

We shall first establish the "only if" part of Claim (i) as well as the uniqueness of Claim (ii). For these purposes, assume that there exists a pair $\left(\mathcal{B}_{2}, \mathcal{A}\right)$ of a $\mathrm{DHO} \mathcal{B}_{2}$ and a DHO $\mathcal{A}$ of rank $n$ and $n+1$ respectively which satisfies

$$
\begin{aligned}
& \mathbf{U}(\mathcal{A}) \subseteq U, B_{2} \in \mathcal{B}_{2}, A_{2} \in \mathcal{A}, \mathcal{B}_{1} \text { and } \mathcal{B}_{2} \text { are subDHOs of } \mathcal{A} \text { with } \\
& \mathcal{A}=\mathcal{B}_{1} \sqcup \mathcal{B}_{2} \text {. }
\end{aligned}
$$

We also fix a complement $Y_{1}$ above and denote by $A_{1}$ the unique member of $\mathcal{A}$ containing $B_{1}$. Then $A_{1} \cap A_{2}$ is a 1-dimensional subspace of $A_{2}$, which is not contained in $B_{2}$ nor $B_{1}$, because of the remarks in Section 1.2. We denote by $a_{12}$ the unique nonzero vector in $A_{1} \cap A_{2}$. Thus $A_{j}=\left\langle a_{12}, B_{j}\right\rangle$ for $j=1$ and $j=2$.

We shall show that we can parametrize the members of $\mathcal{A}\left(\mathcal{B}_{1}\right), \mathcal{B}_{2}$ and $\mathcal{A}\left(\mathcal{B}_{2}\right)$ by $B_{2}$ so that each of them are uniquely described by the linear system $\mathcal{L}_{B_{2}}\left(\mathcal{B}_{1}, Y_{1}\right)$ (with given parametrization by $B_{2}$ ). This shows the uniqueness in Claim (ii). During this procedure, we shall also show that equation (3) holds for all $d \in B_{2}^{\times}$, $x_{1}, x_{2} \in B_{2}$. This establishes the "only if" part of Claim (i).

From Proposition 1.2 (2), the ambient space $\mathbf{U}(\mathcal{A})$ of $\mathcal{A}$ is a sum of $A_{2}$ and $\mathbf{U}\left(\mathcal{B}_{1}\right)$. Then assumptions (u) and (s1) above implies the following direct sum 


However, this implies that $A_{1}(c) \cap A_{2}\left(d_{1}\right)=\left\langle a\left(c, d_{1}\right)\right\rangle=\left\langle a\left(c, d_{2}\right)\right\rangle=A_{1}(c) \cap$ $A_{2}\left(d_{2}\right)$ by equation (8), which contradicts the condition (A3) for a DHO $\mathcal{A}$. Thus for each $c \in B_{2}$ the map sending $d \in B_{2}$ to $x(c, d)$ is injective and so bijective onto $B_{1}$. As for the latter property $x(c, 0)=0$, observe that $A_{1}(c) \cap A_{2}(0)=$ $A_{1}(c) \cap A_{2}=\left\langle a_{12}+c\right\rangle$. Then equation (8) implies that $a(c, 0)=a_{12}+c+$ $x(c, 0)+x(c, 0) L_{1}(c)=a_{12}+c$. Thus we have $x(c, 0)+x(c, 0) L_{1}(c)=0$ with $x(c, 0) \in B_{1}$ and $x(c, 0) L_{1}(c) \in Y_{1}$. Then the direct sum decomposition $B_{1} \oplus Y_{1}$ implies that $x(c, 0)=0$.

Next we claim that

$$
x(0, d)=\kappa_{d} \text { and } d \phi_{d}=0 \text { for each } d \in B_{2} .
$$

For $0 \neq d \in B_{2}$, we have chosen $A_{2}(d) \in \mathcal{A}\left(\mathcal{B}_{2}\right) \backslash\left\{A_{2}\right\}$ so that $A_{2} \cap A_{2}(d)=$ $B_{2} \cap B_{2}(d)=\langle d\rangle$. From equation (10), this implies $d=c+(x(0, d)+x(c, d))+$ $x(c, d) L_{1}(c)$ for some $c \in B_{2}$. As $c+d \in B_{2}, x(0, d)+x(c, d) \in B_{1}$ and $x(c, d) L_{1}(c) \in Y_{1}$, the direct sum decomposition $B_{2} \oplus B_{1} \oplus Y_{1}$ implies that $c=d, x(0, d)=x(d, d)$, and $x(d, d) L_{1}(d)=0$. As $d \neq 0$, the kernel of the linear map $L_{1}(d)$ is a 1-dimensional subspace of $B_{1}$ generated by $\kappa_{d}$ (see Section 1.5). By the conclusion in the above paragraph, $x(d, d) \neq x(0,0)=0$ for $d \neq 0$. Thus we have $\kappa_{d}=x(d, d)=x(0, d)$ for every $0 \neq d \in B_{2}$. Moreover, $d \phi_{d}=x(d, d)+x(0, d)=0$ for all $d \in B_{2}$.

In particular, we have

$$
c \phi_{d}=x(c, d)+\kappa_{d} \text { and } c \psi_{d}=\left(c \phi_{d}+\kappa_{d}\right) L_{1}(c) \text { for all } c, d \in B_{2} .
$$

Now we shall establish the linearity of $\phi_{d}$ and $\psi_{d}$ for $d \in B_{2}$. We use the fact that $B_{2}(d)\left(d \in B_{2}\right)$ has a structure of a subspace. In view of the shapes of vectors in $B_{2}(d)$ given in (10), this implies that for any vectors $c_{1}$ and $c_{2}$ of $B_{2}$ there is a vector $c \in B_{2}$ satisfying the following equation:

$$
\begin{aligned}
& c_{1}+\left(x(0, d)+x\left(c_{1}, d\right)\right)+x\left(c_{1}, d\right) L_{1}\left(c_{1}\right) \\
+ & c_{2}+\left(x(0, d)+x\left(c_{2}, d\right)\right)+x\left(c_{2}, d\right) L_{1}\left(c_{2}\right) \\
= & c+(x(0, d)+x(c, d))+x(c, d) L_{1}(c) .
\end{aligned}
$$

Again the direct sum decomposition of $B_{2} \oplus B_{1} \oplus Y_{1}$ implies that

$$
\begin{aligned}
c & =c_{1}+c_{2}, \\
\left(x(0, d)+x\left(c_{1}, d\right)\right)+\left(x(0, d)+x\left(c_{2}, d\right)\right) & =x(0, d)+x\left(c_{1}+c_{2}, d\right), \text { and } \\
x\left(c_{1}, d\right) L_{1}\left(c_{1}\right)+x\left(c_{2}, d\right) L_{1}\left(c_{2}\right) & =x\left(c_{1}+c_{2}, d\right) L_{1}\left(c_{1}+c_{2}\right) .
\end{aligned}
$$

The second and the last equations above respectively show the linearity of $\phi_{d}$ and $\psi_{d}\left(d \in B_{2}\right)$. 
Finally we shall show equation (3). Notice that

$$
d \psi_{d}=\left(d \phi_{d}+\kappa_{d}\right) L_{1}(d)=\left(0+\kappa_{d}\right) L_{1}(d)=0
$$

by the above relation between $\phi_{d}$ and $\psi_{d}$, the property $d \phi_{d}=0$, and the definition of $\kappa_{d}$. Take any $c \in B_{2}$. As $\psi_{d}$ is a linear map with $d \psi_{d}=0$, we have

$$
\begin{aligned}
0 & =d \psi_{d}=(d+c+c) \psi_{d}=(d+c) \psi_{d}+c \psi_{d} \\
& =\left((d+c) \phi_{d}+\kappa_{d}\right) L_{1}(d+c)+\left(c \phi_{d}+\kappa_{d}\right) L_{1}(c) \\
& =\left(c \phi_{d}+\kappa_{d}\right)\left(L_{1}(d+c)+L_{1}(c)\right)
\end{aligned}
$$

by the above relation between $\phi_{d}$ and $\psi_{d}$ as well as the linearity of $\phi_{d}$ and the property $d \phi_{d}=0$. As $c \phi_{d}+\kappa_{d}=x(c, d)$, the injectivity of the map $B_{2} \ni$ $d \mapsto c \phi_{d}+\kappa_{d} \in B_{1}\left(c \in B_{2}\right)$ we established as the first claim above implies that $c \phi_{d}+\kappa_{d} \neq c \phi_{0}+\kappa_{0}=x(c, 0)=0$ if $d \neq 0$. Hence the above equation implies that $c \phi_{d}+\kappa_{d}=\kappa(d+c, c)$ for $d \neq 0$ by the definition of $\kappa(c+d, c)$ (see Section 1.5). Summarizing, we have

$$
\begin{aligned}
x(c, d)+x(0, d) & =c \phi_{d}=\kappa(d+c, c)+\kappa_{d} \text { and } \\
x(c, d) L_{1}(c) & =c \psi_{d}=\kappa(d+c, c) L_{1}(c)=\kappa(d+c, c) L_{1}(d+c)
\end{aligned}
$$

for all $c \in B_{2}$ and $d \in B_{2}^{\times}$. Recall that $b_{1}\{a, b\}=\kappa(a, b)+\kappa(a, b) L_{1}(a)$ (see Section 1.5). Thus we have

$$
b_{1}\{d+x, x\}=\kappa(d+x, x)+\kappa(d+x, x) L_{1}(x)=x \phi_{d}+\kappa_{d}+x \psi_{d}
$$

for all $x \in B_{2}$. In particular, $b_{1}\{d, 0\}=\kappa_{d}$. Now the desired equation (3) follows from the linearity of $\phi_{d}$ and $\psi_{d}$.

Thus we established the "only if" part of Claim (i).

With the above notation, $\mathcal{B}_{2}$ splits over the subspace, say $Y_{2}$, of $B_{1} \oplus Y_{1}$ spanned by $c \phi_{d}+c \psi_{d}=\left(\kappa(d+c, c)+\kappa_{d}\right)+\kappa(d+c, c) L_{1}(c)$ for all $c, d \in B_{2}$, because $Y_{2} \subseteq B_{1} \oplus Y_{1}$ and $B_{2}(d) \cap\left(B_{1} \oplus Y_{1}\right)=\{0\}$ for all $d \in B_{2}$ in view of equation (10). Moreover the linear system for $\mathcal{B}_{2}$ over $Y_{2}$ is $\mathcal{L}_{B_{2}}\left(\mathcal{B}_{2}, Y_{2}\right)=$ $\left\{L_{2}(d) \mid d \in B_{2}\right\}$, where $L_{2}(d)\left(d \in B_{2}\right)$ is given by

$$
c L_{2}(d):=c \phi_{d}+c \psi_{d}=\left(\kappa(d+c, c)+\kappa_{d}\right)+\kappa(d+c, c) L_{1}(c) \quad\left(c \in B_{2}\right) .
$$

As $\kappa(d+c, c)$ for $c \in B_{2}, d \in B_{2}^{\times}$are uniquely determined by the given linear system $\mathcal{L}_{B_{2}}\left(\mathcal{B}_{1}, Y_{1}\right)$ for $\mathcal{B}_{1}$ over $Y_{1}$, this implies that $\mathcal{B}_{2}$ is uniquely determined by $\mathcal{B}_{1}$ (under our choice of $B_{1}, A_{1}, B_{2}, A_{2}$ and a complement $Y_{1}$ ). Furthermore, $\mathcal{A}$ is uniquely determined by the given linear system $\mathcal{L}_{B_{2}}\left(\mathcal{B}_{1}, Y_{1}\right)$, as the members $A_{2}(d)$ are uniquely described by $x(c, d)+x(0, d)=c \phi_{d}=\kappa(c+d, c)+\kappa_{d}$ and $x(c, d) L_{1}(c)=c \psi_{d}=\kappa(c+d, c) L_{1}(c)\left(c, d \in B_{2}\right)$. 

As $c_{1} \neq c_{2}$, this is equivalent to $\varepsilon=0$ and $x$ lies in the 1-dimensional kernel of $L_{1}\left(c_{1}\right)+L_{1}\left(c_{2}\right)$, spanned by $\kappa\left(c_{1}, c_{2}\right)$. Thus $v$ lies in $B_{1}\left(c_{1}\right) \cap B_{1}\left(c_{2}\right)$, which is spanned by $\kappa\left(c_{1}, c_{2}\right)+\kappa\left(c_{1}, c_{2}\right) L_{1}\left(c_{1}\right)$. This verifies that $A_{1}\left(c_{1}\right) \cap$ $A_{1}\left(c_{2}\right)=B_{1}\left(c_{1}\right) \cap B_{1}\left(c_{2}\right)$ is a 1-dimensional subspace spanned by $\kappa\left(c_{1}, c_{2}\right)+$ $\kappa\left(c_{1}, c_{2}\right) L_{1}\left(c_{1}\right)$.

Next we shall examine $A_{2}\left(d_{1}\right) \cap A_{2}\left(d_{2}\right)$ for distinct $d_{1}, d_{2}$ of $B_{2}$ (this is the only case to examine for showing that $\mathcal{B}_{2}$ satisfies condition (A1)). Again any vector $v$ in $A_{2}\left(d_{1}\right) \cap A_{2}\left(d_{2}\right)$ has the following expressions

$$
\begin{aligned}
v & =\varepsilon_{1} a_{12}+c_{1}+\left(\varepsilon_{1} \kappa_{d_{1}}+c_{1} \phi_{d_{1}}\right)+\left(c_{1} \phi_{d_{1}}+\kappa_{d_{1}}\right) L_{1}\left(c_{1}\right) \\
& =\varepsilon_{2} a_{12}+c_{2}+\left(\varepsilon_{2} \kappa_{d_{2}}+c_{2} \phi_{d_{2}}\right)+\left(c_{2} \phi_{d_{2}}+\kappa_{d_{2}}\right) L_{1}\left(c_{2}\right)
\end{aligned}
$$

for some $\varepsilon_{j} \in \mathbb{F}_{2}$ and $c_{j} \in B_{2}(j=1,2)$. As $\left(\varepsilon_{1}+\varepsilon_{2}\right) a_{12} \in\left\langle a_{12}\right\rangle, c_{1}+c_{2} \in B_{2}$, $\varepsilon_{1} \kappa_{d_{1}}+c_{1} \phi_{d_{1}}+\varepsilon_{2} \kappa_{d_{2}}+c_{2} \phi_{d_{2}} \in B_{1}$, and $\left(c_{1} \phi_{d_{1}}+\kappa_{d_{1}}\right) L_{1}\left(c_{1}\right)+\left(c_{2} \phi_{d_{2}}+\kappa_{d_{2}}\right) L_{1}\left(c_{2}\right) \in$ $Y_{1}$, the direct sum decomposition (14) implies that we have

$$
\begin{aligned}
\varepsilon_{1}=\varepsilon_{2}=: \varepsilon ; \quad c_{1}=c_{2}=: c ; \quad \varepsilon \kappa_{d_{1}}+c \phi_{d_{1}}=\varepsilon \kappa_{d_{2}}+c \phi_{d_{2}} \\
\\
\left(c \phi_{d_{1}}+\kappa_{d_{1}}\right) L_{1}(c)=\left(c \phi_{d_{2}}+\kappa_{d_{2}}\right) L_{1}(c) .
\end{aligned}
$$

If $\varepsilon=1$, the third equation reads $\kappa_{d_{1}}+c \phi_{d_{1}}=\kappa_{d_{2}}+c \phi_{d_{2}}$, which is equivalent to $\kappa\left(d_{1}+c, c\right)=\kappa\left(d_{2}+c, c\right)$ by the definition of $\phi_{d}$ for $d=d_{1}, d_{2}$. As $\mathcal{L}_{B_{2}}\left(\mathcal{B}_{1}, Y_{1}\right)$ is a linear system for $\mathcal{B}_{1}$ over $Y_{1}$, the map from $B_{2} \backslash\{c\}$ to $B_{1} \backslash\{0\}$ sending $x$ to $\kappa(x, c)$ is injective (see property (L2) for a linear system, in Section 1.5). Then we have $d_{1}=d_{2}$, which contradicts the choice of $d_{1}$ and $d_{2}$. Thus $\varepsilon=0$ and $c \phi_{d_{1}}=c \phi_{d_{2}}$ from the third equation, and hence

$$
\left(\kappa_{d_{1}}+\kappa_{d_{2}}\right) L_{1}(c)=0
$$

from the last equation with the linearity of $L_{1}(c)$. This equation uniquely determines nonzero $c$ as follows. By the above mentioned injectivity of the map from $B_{2}^{\times}$to $B_{1}^{\times}$sending $x$ to $\kappa(x, 0)=\kappa_{x}$ (corresponding to property (L2) for a linear system, in Section 1.5), there exists a unique nonzero element $d_{3}$ of $B_{2}$ such that $\kappa_{d_{1}}+\kappa_{d_{2}}=\kappa_{d_{3}}$, and then the above equation is equivalent to $\kappa_{d_{3}} L_{1}(c)=0$. (If $c=0$, then we obtain the trivial solution $v=0$.) If $c \neq 0$, it follows from the definition of $\kappa_{c}$ that $\kappa_{c}=\kappa_{d_{3}}$. Thus $c=d_{3}$ from the injectivity of the map $x \mapsto \kappa_{x}$. Hence we conclude that $A_{2}\left(d_{1}\right) \cap A_{2}\left(d_{2}\right)=B_{2}\left(d_{1}\right) \cap B_{2}\left(d_{2}\right)$ is a 1-dimensional subspace spanned by $d_{3}+d_{3} \phi_{d_{1}}+\left(d_{3} \phi_{d_{1}}+\kappa_{d_{1}}\right) L_{1}\left(d_{3}\right)$ for any distinct $d_{1}, d_{2}$ of $B_{2}$, where $d_{3}$ denotes the unique (nonzero) vector of $B_{2}$ with $\kappa_{d_{1}}+\kappa_{d_{2}}=\kappa_{d_{3}}$.

In particular, we have $B_{2}\left(d_{1}\right) \cap B_{2}\left(d_{2}\right) \cap B_{2}\left(d_{2}^{\prime}\right)=\{0\}$ if $d_{1}, d_{2}, d_{2}^{\prime}$ are pairwise distinct from the injectivity of the map $x \mapsto \kappa_{x}$. Thus we already verified that $\mathcal{B}_{2}$ satisfies condition (A2) for a DA as well, and therefore $\mathcal{B}_{2}$ is in fact a DHO. 

(ii) Fix an arbitrary linear bijection $\rho$ from $B_{2}$ onto $B_{1}$. It is straightforward to verify that

$$
Y=Y_{\rho}:=\left\{e+(e) \rho+y \mid e \in B_{2}, y \in Y_{1}\right\}
$$

is a subspace of $\mathbf{U}(\mathcal{A})$ satisfying $Y \cap A_{1}(c)=Y \cap A_{2}(c)=\{0\}$ for all $c \in B_{2}$, in view of the direct sum decomposition $\left\langle a_{12}\right\rangle \oplus B_{2} \oplus B_{1} \oplus Y_{1}$ of $\mathbf{U}(\mathcal{A})$ and the expressions

$$
\begin{aligned}
& A_{1}(c)=\left\{\varepsilon a_{12}+\varepsilon c+x+x L_{1}(c) \mid \varepsilon \in \mathbb{F}_{2}, x \in B_{1}\right\}, \\
& A_{2}(c)=\left\{\varepsilon a_{12}+x^{\prime}+\varepsilon \kappa_{c}+\kappa_{c} L_{1}\left(x^{\prime}\right) \mid \varepsilon \in \mathbb{F}_{2}, x^{\prime} \in B_{2}\right\} .
\end{aligned}
$$

Fix a member $A_{1}(0)=\left\{\varepsilon a_{12}+x \mid x \in B_{1}\right\}$ of $\mathcal{A}$. The following gives the decomposition of a typical vector $v$ of $A_{1}(c)$ or $A_{2}(c)$ in the form $v=v_{1}+v_{2}$ with $v_{1} \in A_{1}(0)$ and $v_{2} \in Y$ :

- for $v=\varepsilon a_{12}+\varepsilon c+x+x L_{1}(c)$ of $A_{1}(c)\left(\varepsilon \in \mathbb{F}_{2}, x \in B_{1}\right)$, we have $v_{1}=\varepsilon a_{12}+(x+\varepsilon(c) \rho)$ and $v_{2}=\varepsilon c+\varepsilon(c) \rho+x L_{1}(c)$;

- for $v=\varepsilon a_{12}+x^{\prime}+\varepsilon \kappa_{c}+\kappa_{c} L_{1}\left(x^{\prime}\right)$ of $A_{2}(c)\left(\varepsilon \in \mathbb{F}_{2}, x^{\prime} \in B_{2}\right)$, we have $v_{1}=\varepsilon a_{12}+\left(\varepsilon \kappa_{c}+\left(x^{\prime}\right) \rho\right)$ and $v_{2}=x^{\prime}+\left(x^{\prime}\right) \rho+\kappa_{c} L_{1}\left(x^{\prime}\right)$.

Then, setting $y=x+\varepsilon(c) \rho \in B_{1}$ (resp. $y=\varepsilon \kappa_{c}+\left(x^{\prime}\right) \rho \in B_{1}$ ) in the above expression of $v_{2}$ for $v \in A_{1}(c)$ (resp. $A_{2}(c)$ ), the linear maps $L(c, 0)$ and $L(c, 1)$ sending $v_{1}$ to $v_{2}$ can be described as in equation (4).

(iii) Assume that $\mathcal{A}$ is bilinear with respect to $Y_{\rho}$ for a linear bijection $\rho$ from $B_{2}$ onto $B_{1}$. Then there is a permutation $\pi$ on $B_{2} \times \mathbb{F}_{2}=\{(c, \delta) \mid c \in$ $\left.B_{2}, \delta \in \mathbb{F}_{2}\right\}$ (with componentwise addition) such that

$$
L\left(\left(c_{1}, \delta_{1}\right)^{\pi}\right)+L\left(\left(c_{2}, \delta_{2}\right)^{\pi}\right)=L\left(\left(c_{1}+c_{2}, \delta_{1}+\delta_{2}\right)^{\pi}\right)
$$

for all $\left(c_{1}, \delta_{1}\right),\left(c_{2}, \delta_{2}\right) \in B_{2} \times \mathbb{F}_{2}$. (We should mention the permutation $\pi$ here, because we already fix an ordering on $B_{2}$ so that equation (15) is satisfied for $\mathcal{L}_{B_{2}}\left(\mathcal{B}_{1}, Y_{1}\right)$.) Then for any $c_{1}, c_{2}$ of $B_{2}$ we have

$$
\begin{aligned}
L\left(\left(c_{1}, 0\right)\right)+L\left(\left(c_{2}, 0\right)\right) & =L\left(\left(\left(c_{1}, 0\right)^{\pi^{-1}}\right)^{\pi}\right)+L\left(\left(\left(c_{2}, 0\right)^{\pi^{-1}}\right)^{\pi}\right) \\
& \left.=L\left(\left(\left(c_{1}, 0\right)^{\pi^{-1}}+\left(c_{2}, 0\right)^{\pi^{-1}}\right)^{\pi}\right)\right) .
\end{aligned}
$$

We set $(c, \delta):=\left(\left(c_{1}, 0\right)^{\pi^{-1}}+\left(c_{2}, 0\right)^{\pi^{-1}}\right)^{\pi}$. Take any $\varepsilon a_{12}+y$ of $A_{1}(0)$ with $\varepsilon \in \mathbb{F}_{2}, y \in B_{1}$. It follows from equation (17) together with (4) that

$$
\begin{aligned}
& \left(\varepsilon a_{12}+y\right)\left(L\left(c_{1}, 0\right)+L\left(c_{2}, 0\right)\right) \\
& =\varepsilon\left(c_{1}+c_{2}\right)+\varepsilon\left(c_{1}+c_{2}\right) \rho \\
& \quad+\left\{y\left(L_{1}\left(c_{1}\right)+L_{1}\left(c_{2}\right)\right)+\varepsilon\left(\left(c_{1}\right) \rho L_{1}\left(c_{1}\right)+\left(c_{2}\right) \rho L_{1}\left(c_{2}\right)\right)\right\}
\end{aligned}
$$


This equals to $\left(\varepsilon a_{12}+y\right) L(c, \delta)$ for all $\varepsilon \in \mathbb{F}_{2}, y \in B_{1}$. Suppose $\delta=1$. Putting $\varepsilon=0$ and then comparing the component in $B_{2}$ of $(y)\left(L\left(c_{1}, 0\right)+\right.$ $\left.L\left(c_{2}, 0\right)\right)$ obtained above with that of $(y) L(c, 1)$ in equation (4), we have $0=(y) \rho^{-1}$ for all $y \in B_{1}$, which is a contradiction. Thus $\delta=0$. Then, putting $\varepsilon=1$, we have $c=c_{1}+c_{2}$ by comparing the component in $B_{2}$ of $\left(a_{12}+y\right) L(c, 0)$ in equation (4) with that of $\left(a_{12}+y\right)\left(L\left(c_{1}, 0\right)+L\left(c_{2}, 0\right)\right)$ in the above equation. Hence we established $L\left(c_{1}, 0\right)+L\left(c_{2}, 0\right)=L\left(c_{1}+c_{2}, 0\right)$ for all $c_{1}, c_{2} \in B_{2}$.

Using the bilinearity of $\left\{L_{1}(t) \mid t \in B_{2}\right\}$, we have the following equation for any $\varepsilon \in \mathbb{F}_{2}$ and $y \in B_{1}$ from equation (4):

$$
\begin{aligned}
&\left(\varepsilon a_{12}+y\right)\left(L\left(c_{1}+c_{2}, 0\right)+L\left(c_{1}, 0\right)+L\left(c_{2}, 0\right)\right) \\
&= y\left\{L_{1}\left(c_{1}+c_{2}\right)+L_{1}\left(c_{1}\right)+L_{1}\left(c_{2}\right)\right\} \\
& \quad \quad+\varepsilon\left\{\left(c_{1}+c_{2}\right) \rho L_{1}\left(c_{1}+c_{2}\right)+\left(c_{1}\right) \rho L_{1}\left(c_{1}\right)+\left(c_{2}\right) \rho L_{1}\left(c_{2}\right)\right\} \\
&= \\
& \quad \varepsilon\left\{\left(c_{1}\right) \rho L_{1}\left(c_{2}\right)+\left(c_{2}\right) \rho L_{1}\left(c_{1}\right)\right\}
\end{aligned}
$$

As $L\left(c_{1}+c_{2}, 0\right)=L\left(c_{1}, 0\right)+L\left(c_{2}, 0\right)$, we have equation (5) by putting $\varepsilon=1$.

Assume that equation (5) is satisfied. Then the above calculation shows that $L\left(c_{1}, 0\right)+L\left(c_{2}, 0\right)=L\left(c_{1}+c_{2}, 0\right)$ for all $c_{1}, c_{2} \in B_{2}$. We shall verify that the following equation holds for any $c_{1}, c_{2}$ of $B_{2}$ :

$$
L\left(c_{1}, 1\right)+L\left(c_{2}, 1\right)=L\left(\left(\kappa_{c_{1}}+\kappa_{c_{2}}\right) \rho^{-1}, 0\right) .
$$

We obtain the following equation for $\varepsilon \in \mathbb{F}_{2}, y \in B_{1}$, using equation (4), the linearity of $\rho$ and the bilinearity of $L_{1}(c)\left(c \in B_{2}\right)$ :

$$
\begin{aligned}
& \left(\varepsilon a_{12}+y\right)\left(L\left(c_{1}, 1\right)+L\left(c_{2}, 1\right)\right) \\
& =\left\{\left(\varepsilon \kappa_{c_{1}}+y\right)+\left(\varepsilon \kappa_{c_{2}}+y\right)\right\} \rho^{-1}+\varepsilon\left(\kappa_{c_{1}}+\kappa_{c_{2}}\right) \\
& \quad+\varepsilon\left\{\kappa_{c_{1}} L_{1}\left(\kappa_{c_{1}} \rho^{-1}\right)\right. \\
& \left.+\kappa_{c_{2}} L_{1}\left(\kappa_{c_{2}} \rho^{-1}\right)\right\} \\
& \quad+\left\{\kappa_{c_{1}} L_{1}\left(y \rho^{-1}\right)+\kappa_{c_{2}} L_{1}\left(y \rho^{-1}\right)\right\}
\end{aligned}
$$

At the last line in the above equation, observe that

$$
\left(\kappa_{c_{1}}+\kappa_{c_{2}}\right) L_{1}\left(\left(\kappa_{c_{1}}+\kappa_{c_{2}}\right) \rho^{-1}\right)=\kappa_{c_{1}} L_{1}\left(\kappa_{c_{1}} \rho^{-1}\right)+\kappa_{c_{2}} L_{1}\left(\kappa_{c_{2}} \rho^{-1}\right)
$$

by the linearity of $\rho^{-1}$, the bilinearity of $L_{1}(c)\left(c \in B_{2}\right)$ and equation (5) applied to $(c, d)=\left(\kappa_{c_{2}}, \kappa_{c_{1}} \rho^{-1}\right)$. Similarly, at the last line in the above equation we have

$$
\begin{aligned}
\kappa_{c_{1}} L_{1}\left(y \rho^{-1}\right)+\kappa_{c_{2}} L_{1}\left(y \rho^{-1}\right) & =y L_{1}\left(\kappa_{c_{1}} \rho^{-1}\right)+y L_{1}\left(\kappa_{c_{2}} \rho^{-1}\right) \\
& =y L_{1}\left(\left(\kappa_{c_{1}}+\kappa_{c_{2}}\right) \rho^{-1}\right) .
\end{aligned}
$$


Then it follows from equation (4) that we have

$$
\left(\varepsilon a_{12}+y\right)\left(L\left(c_{1}, 1\right)+L\left(c_{2}, 1\right)+L\left(\left(\kappa_{c_{1}}+\kappa_{c_{2}}\right) \rho^{-1}, 0\right)\right)=0
$$

for any $\varepsilon \in \mathbb{F}_{2}$ and $y \in B_{2}$, as desired.

It remains to show that $\mathcal{B}_{1}$ is isomorphic to $\mathcal{B}_{2}$, if equation (5) is satisfied. Recall that $\mathcal{B}_{1}$ consists of $B_{1}(c)=\left\{x+x L_{1}(c) \mid x \in B_{1}\right\}$ for $c \in B_{2}$, while $\mathcal{B}_{2}$ consists of $B_{2}(d)=\left\{c+\kappa_{d} L_{1}(c) \mid c \in B_{2}\right\}$ for $d \in B_{2}$. Assume that equation (5) holds for a linear bijection $\rho$ from $B_{2}$ to $B_{1}$. Define a linear map $\bar{\rho}$ from $\mathbf{U}\left(\mathcal{B}_{2}\right)=B_{2} \oplus Y_{1}$ to $\mathbf{U}\left(\mathcal{B}_{1}\right)=B_{1} \oplus Y_{1}$ by

$$
(c+y) \bar{\rho}:=(c \rho)+y, \text { for } c \in B_{2}, y \in Y_{1} .
$$

Then a typical vector $c+\kappa_{d} L_{1}(c)\left(c \in B_{2}\right)$ of $B_{2}(d)$ is mapped by $\bar{\rho}$ to (c) $\rho+\kappa_{d} L_{1}(c)$, which is equal to

$$
(c) \rho+\kappa_{d} L_{1}(c)=(c) \rho+(c) \rho L_{1}\left(\left(\kappa_{d}\right) \rho^{-1}\right)
$$

by equation (5). As $c$ runs over $B_{2}$, this vector runs over the member $B_{1}\left(\left(\kappa_{d}\right) \rho^{-1}\right)$. Thus $B_{2}(d) \bar{\rho}=B_{1}\left(\kappa_{d} \rho^{-1}\right)$ for every $d \in B_{2}$, and hence $\bar{\rho}$ induces an isomorphism from $\mathcal{B}_{2}$ to $\mathcal{B}_{1}$.

\section{Some examples}

In this section, we discuss several examples of Theorem 1.3, specifically those of Corollary 1.4.

First, we treat four known infinite families $\mathcal{H}_{n}\left(\mathbb{F}_{2}\right), \mathcal{D}_{n}\left(\mathbb{F}_{2}\right), \mathcal{V}_{n}\left(\mathbb{F}_{2}\right)$ and $\mathcal{T}_{n}\left(\mathbb{F}_{2}\right)$ of DHOs over $\mathbb{F}_{2}$, defined for each $n \geq 3$, with ambient spaces of dimension $n(n+1) / 2$. (See [7] for a uniform description of them.) The former two are bilinear over some complements, but the latter two are not. It is immediate to verify whether each of the latter two families satisfies the criterion in Theorem 1.3(i), as the intersection of two members are already calculated. It turns out that none of them cannot be extended to a DHO with conditions in Theorem 1.3(i). See Section 3.1. We shall show that each of the former two can be extended to a DHO in the same family. See Section 3.2.

Then we discuss a family of DHOs $\mathcal{Y}^{\sigma, \tau}$ of rank $n$ over $\mathbb{F}_{2}$ with ambient space of dimension $2 n$ or $2 n-1$ parametrized by a pair $(\sigma, \tau)$ of generators $\sigma$ and $\tau$ of the Galois group of $\mathbb{F}_{2^{n}}$. It is extended to be a DHO $\mathcal{A}$ with conditions in Theorem 1.3(i), as it is bilinear over a complement. We determine the complements to the extension $\mathcal{A}$ over which $\mathcal{A}$ is bilinear, if they are of shape $Y_{\rho}$ with automorphisms $\rho$ of the underlying vector space of $\mathbb{F}_{2^{n}}$. See Section 3.3. 


\subsection{Huybrechts DHOs and Buratti-Del Fra DHOs}

The Huybrechts DHO $\mathcal{H}_{n}\left(\mathbb{F}_{2}\right)$ has the ambient space $F \oplus K=\{(x, y) \mid x \in$ $F, y \in K\}$, where $F$ is the underlying vector space of $\mathbb{F}_{2^{n}}$ and $K=F \wedge F$, the alternating square tensor product of $F$. Notice that $F \wedge F$ is identical to the factor space $(F \otimes F) / W$, where $W$ is a subspace of the tensor product $F \otimes F$ spanned by $x \otimes y+y \otimes x$ and $x \otimes x$ for all $x, y \in F$. The image $(x \otimes y)+W$ of $x \otimes y$ in $(F \otimes F) / W=F \wedge F$ is denoted $x \wedge y$ for short. The DHO $\mathcal{H}_{n}\left(\mathbb{F}_{2}\right)$ splits over $Y_{1}:=\{(0, y) \mid y \in K\}$. We adopt $F$ to parametrize the members of $\mathcal{B}_{1}:=\mathcal{H}_{n}\left(\mathbb{F}_{2}\right)$ with $B_{1}(0)=B_{1}:=\{(x, 0) \mid x \in F\}$. The linear system $\mathcal{L}_{F}\left(\mathcal{H}_{n}\left(\mathbb{F}_{2}\right), K\right)$ is given as the collection of linear maps $L_{1}(t)(t \in F)$ sending each vector $(x, 0)$ of the member $B_{1}(0)$ to $(x, 0) L_{1}(t)=(0, x \wedge t)$. In particular, $\mathcal{B}_{1}$ is bilinear over $Y_{1}$.

Thus we can apply Corollary 1.4(i) to $\mathcal{B}_{1}=\mathcal{H}_{n}\left(\mathbb{F}_{2}\right)$ to conclude that there is a unique DHO $\mathcal{A}$ (called the extension $\mathcal{A}\left(B_{1}, Y_{1}\right)$ in Section 1.7) of rank $n+1$ over $\mathbb{F}_{2}$ as well as its subDHO $\mathcal{B}_{2}$ of corank 1 such that the following holds:

$\mathbf{U}(\mathcal{A})=\mathbb{F}_{2} \oplus F \oplus F \oplus K=\left\{(\varepsilon ; z, x, y) \mid \varepsilon \in \mathbb{F}_{2}, x, z \in F, y \in K\right\}$, where $\mathbf{U}\left(\mathcal{B}_{1}\right)$ corresponds to $\{(0 ; 0, x, y) \mid x \in F, y \in K\}$, where $B_{1}=\{(0 ; 0, x, 0) \mid x \in F\}$ and $B_{2}:=\{(0 ; z, 0,0) \mid z \in F\} \in \mathcal{B}_{2}$, $A_{1}:=\left\langle a_{12}, B_{1}\right\rangle \in \mathcal{A}$ and $A_{2}:=\left\langle a_{12}, B_{2}\right\rangle \in \mathcal{A}$ for $a_{12}:=(1 ; 0,0,0)$, and $\mathcal{A}$ is a disjoint union of subDHOs $\mathcal{B}_{1}$ and $\mathcal{B}_{2}$.

Explicitly, $\mathcal{A}=\left\{A_{1}(t), A_{2}(t) \mid t \in F\right\}$, where $A_{1}(t)=\left\{(\varepsilon, \varepsilon t, x, x \wedge t) \mid \varepsilon \in \mathbb{F}_{2}, x \in\right.$ $F\}$ and $A_{2}(t)=\left\{(\varepsilon, z, \varepsilon t, z \wedge t) \mid \varepsilon \in \mathbb{F}_{2}, z \in F\right\}$. Moreover, $\mathcal{B}_{2}=\left\{B_{2}(t) \mid t \in F\right\}$ with $B_{2}(t)=\{(0 ; z, 0, z \wedge t) \mid z \in F\}$. It follows from Proposition 1.2(3) that the DHO $\mathcal{A}$ is simply connected, as $\mathcal{B}_{1}=\mathcal{H}_{n}\left(\mathbb{F}_{2}\right)$ is simply connected. The dimension of the ambient space of $\mathcal{A}$ is $(n(n+1) / 2)+(n+1)$.

Observe that $(0 ; 0, x, 0) L_{1}(x)=(0 ; 0,0, x \wedge x)=0$ and thus $\kappa_{x}=(0 ; 0, x, 0)$ for all $x \in F$. Hence $L_{1}$, regarded as a bilinear map $L_{1}$ from $B_{1} \times F$ to $Y_{1}$, is alternating. The functions $L_{2}(t)(t \in F)$ in Corollary 1.4(i) for $\mathcal{B}_{2}$ over $Y_{1}$ sends $(0 ; z, 0,0) \in B_{2}$ to $(0 ; 0, t, 0) L_{1}(z)=(0 ; 0,0, z \wedge t)$. Identifying elements $(0 ; 0, x, 0) \in B_{1}$ and $(0 ; z, 0,0) \in B_{2}$ with elements $z$ and $x$ in $F$, respectively, this implies that the maps $L_{2}(t)$ and $L_{1}(t)(t \in F)$ are identical to the map sending $x \in F$ to $x \wedge t \in K$. Thus, under the above identifications, the second DHO $\mathcal{B}_{2}$ is identical to $\mathcal{B}_{1}=\mathcal{H}_{n}\left(\mathbb{F}_{2}\right)$. The DHO $\mathcal{A}$ corresponds to the extension $\overline{\mathcal{S}}$ of $\mathcal{H}_{n}\left(\mathbb{F}_{2}\right)=\mathcal{S}_{\beta}$ for the symmetric bilinear map $\beta(x, t)=x \wedge t(x, t \in F)$ in the sense of [1, Section 5].

We shall verify that $\mathcal{A}$ is in fact isomorphic to $\mathcal{H}_{n+1}\left(\mathbb{F}_{2}\right)$ (with ambient space of dimension $(n+1)(n+2) / 2)$. As $\mathcal{A}$ is a unique DHO satisfying the conditions given in Corollary 1.4(i), it suffices to verify that $\mathcal{H}_{n+1}\left(\mathbb{F}_{2}\right)$ satisfies these 



Corollary 1.4(i):

$$
\begin{aligned}
& A_{1}(c):=\left\{(\varepsilon ; \varepsilon c, x, x L(c)) \mid \varepsilon \in \mathbb{F}_{2}, x \in F\right\}, \\
& A_{2}^{\prime}(d):=\left\{\left(\varepsilon ; z, \varepsilon \kappa_{d}, \kappa_{d} L(z)\right) \mid \varepsilon \in \mathbb{F}_{2}, z \in F\right\} .
\end{aligned}
$$

The second subDHO $\mathcal{B}_{2}$ is a collection of hyperplanes $B_{2}^{\prime}(d):=\left\{\left(0 ; z, 0, \kappa_{d} L(z)\right) \mid\right.$ $z \in F\}$ of $A_{2}^{\prime}(d), d \in F$.

For convenience, we now change the parametrization to the members $A_{2}^{\prime}(d)$ $(d \in F)$. Recall that the map $\kappa: F \ni x \mapsto \kappa_{x} \in F$ is a bijection with $\kappa_{0}=0$ (see Section 1.5). Thus we may rearrange $A_{2}(d):=A_{2}^{\prime}\left(\kappa^{-1}(d)\right)$ and accordingly $B_{2}(d):=B_{2}^{\prime}\left(\kappa^{-1}(d)\right)(d \in F)$. Then for $d \in F$

$$
\begin{aligned}
& A_{2}(d)=\left\{(\varepsilon ; z, \varepsilon d, d L(z)) \mid \varepsilon \in \mathbb{F}_{2}, z \in F\right\} \text { and } \\
& B_{2}(d)=\{(0 ; z, 0, d L(z)) \mid z \in F\} .
\end{aligned}
$$

The members $B_{1}(c)=\{(0 ; 0, x, x L(c)) \mid x \in F\}(c \in F)$ of $\mathcal{B}_{1}$ are copies of the members $X(c)=\left\{(x, x L(c))=\left(x, x^{\sigma} c+x c^{\tau}\right) \mid x \in F\right\}(c \in F)$ of $\mathcal{Y}^{\sigma, \tau}$, while the members $B_{2}^{\prime}(c)=\{(0 ; x, 0, c L(x)) \mid x \in F\}$ of $\mathcal{B}_{2}$ are copies of the members $X^{\prime}(c)=\left\{\left(x, x L^{(\tau, \sigma)}(c)\right) \mid x \in F\right\}$ of $\mathcal{Y}^{\tau, \sigma}$, because $x L^{(\tau, \sigma)}(c)=$ $c^{\sigma} x+c x^{\tau}=c L^{(\sigma, \tau)}(x)$ for $x \in F$. Thus we conclude that $\mathcal{A}$ is a disjoint union of $\mathcal{B}_{1} \cong \mathcal{Y}^{\sigma, \tau}$ and $\mathcal{B}_{2} \cong \mathcal{Y}^{\tau, \sigma}$.

If $\tau \neq \sigma^{-1}$ (resp. $\tau=\sigma^{-1}$ ), we have $\mathbf{U}(\mathcal{A})=\mathbb{F}_{2} \oplus F \oplus F \oplus F$ of dimension $3 n+1$ (resp. $\mathbf{U}(\mathcal{A})$ is a hyperplane of $\mathbb{F}_{2} \oplus F \oplus F \oplus F$ ). If, furthermore, $\mathcal{Y}^{\sigma, \tau}$ is simply connected, it follows from Proposition 1.2(3) that $\mathcal{A}$ is simply connected as well. This DHO $\mathcal{A}$, the extension of $\mathcal{B}_{1}=\mathcal{Y}^{\sigma, \tau}$ in the sense of Section 1.7, was constructed first in [1, Section 5] for the cases $\tau=\sigma$ and $\tau=\sigma^{-1}$, and generalized by Taniguchi in [4] for the other cases. However, the authors of [1] and [4] do not work in the broader context of disjoint unions of subDHOs nor mention the uniqueness in Corollary 1.4.

Notice that for any linear bijection $\lambda$ on $F, \mathcal{A}$ splits over $Y_{\lambda}:=\left\{\left(0 ; x, x^{\lambda}, y\right) \mid\right.$ $x, y \in F\}$. (This corresponds to the complement $Y_{\rho}$ in Corollary 1.4(ii),(iii) with $\rho: B_{2} \ni(0 ; x, 0,0) \mapsto\left(0 ; 0, x^{\lambda}, 0\right) \in B_{1}$. We shall determine all $\lambda$ for which $\mathcal{A}$ is bilinear over $Y_{\lambda}$. (The author does not know whether $\mathcal{A}$ is bilinear over another complement $Y_{\lambda}^{\prime}:=\{(\operatorname{tr}(y) ; x, x, y) \mid x, y \in F\}$. We can verify that $Y_{\lambda}^{\prime}$ is a complement to $\mathcal{A}$ by the arguments similar to those in the observations about complements of $\mathcal{Y}^{\sigma, \tau}$.)

Assume that the linear system $\left\{L(c, \delta) \mid c \in F, \delta \in \mathbb{F}_{2}\right\}$ for $\mathcal{A}$ over $Y=Y_{\lambda}$ (given in Corollary 1.4(iii)) is bilinear. Then we have $\left(c^{\lambda}\right) L(x)=\left(x^{\lambda}\right) L(c)$ for any $c, x \in F$ by equation (5) in Corollary 1.4(ii). Furthermore, it follows from the last remark in Corollary 1.4 (iii) that $\mathcal{B}_{1} \cong \mathcal{Y}^{\sigma, \tau}$ is isomorphic to $\mathcal{B}_{2} \cong \mathcal{Y}^{\tau, \sigma}$. 
As we reviewed in the above paragraph, this happens only when $\tau=\sigma^{-1}$ or $\tau=\sigma$. Correspondingly, the above equation $\left(c^{\lambda}\right) L(x)=\left(x^{\lambda}\right) L(c)$ reads

$$
\begin{aligned}
\left(c^{\lambda}\right)^{\sigma} x+c^{\lambda} x^{\sigma^{-1}} & =\left(x^{\lambda}\right)^{\sigma} c+x^{\lambda} c^{\sigma^{-1}} & \text { if } \tau & =\sigma^{-1}, \\
\left(c^{\lambda}\right)^{\sigma} x+c^{\lambda} x^{\sigma} & =\left(x^{\lambda}\right)^{\sigma} c+x^{\lambda} c^{\sigma} & & \text { if } \tau=\sigma .
\end{aligned}
$$

We consider the former case where $\sigma^{-1}=\tau$. We first claim that any bijective linear map $\lambda$ on $F$ satisfying equation (21) for all $x, c \in F$ is given by $x^{\lambda}=$ $k x^{\sigma^{-1}}(x \in F)$ for a nonzero element $k$ of $F$.

This claim is verified as follows. Equation (21) implies that $c^{\lambda \sigma} x+x^{\lambda \sigma} c=$ $\left(c^{\lambda \sigma} x+x^{\lambda \sigma} c\right)^{\sigma^{-1}}$, whence $\epsilon(c, x):=c^{\lambda \sigma} x+x^{\lambda \sigma} c$ lies in $\mathbb{F}_{2}$, as $\sigma$ is a generator of $\operatorname{Gal}\left(F / \mathbb{F}_{2}\right)$. By the bijectivity of $\lambda$, there is an element $c_{0} \in F$ with $c_{0}^{\lambda}=1$. Then $c_{0} \neq 0$ and $k:=c_{0}^{-\sigma^{-1}}$ satisfies $x^{\lambda}=k\left(x^{\sigma^{-1}}+\epsilon(x)\right)$ for all $x \in F$, where we denote $\epsilon(x):=\epsilon\left(c_{0}, x\right)\left(\in \mathbb{F}_{2}\right)$. As $\lambda, \sigma^{-1}$ and the multiplication by $k$ are linear maps, the map $\epsilon$ sending $x \in F$ to $\epsilon(x) \in \mathbb{F}_{2}$ is linear. In particular, the kernel of $\epsilon$ is of dimension at least $n-1 \geq 2$. On the other hand, putting $y^{\lambda}=k\left(y^{\sigma^{-1}}+\epsilon(y)\right)$ for $y=c, x$ into equation (21), we obtain $\epsilon(c)\left(k^{\sigma} x+k x^{\sigma^{-1}}\right)=\epsilon(x)\left(k^{\sigma} c+k c^{\sigma^{-1}}\right)$ for all $c, x \in F$. Suppose there is $c \in F$ with $\epsilon(c)=1$. Then any $x$ in the kernel of $\epsilon$ satisfies $k^{\sigma} x+k x^{\sigma^{-1}}=0$, whence $x=0$ or $x=k^{-\sigma}$ by the injectivity of $\sigma-\operatorname{id}_{F}$. This contradicts the above remark on the dimension of the kernel of $\epsilon$. Hence we have $x^{\lambda}=k x^{\sigma^{-1}}$ for all $x \in F$. Conversely, for any nonzero element $k$ of $F$, the bijective linear map $\lambda$ defined by $x^{\lambda}=k x^{\sigma^{-1}}(x \in F)$ satisfies equation (21) for all $x, c \in F$.

Now we fix a nonzero element $k$ of $F$. We denote by $\lambda$ a bijective linear map on $F$ defined by $x^{\lambda}=k x^{\sigma^{-1}}(x \in F)$. With some calculations, we can verify that equations (4) in Corollary 1.4(ii) read

$$
\begin{gathered}
(\varepsilon ; 0, y, 0) L(c, 0)=\varepsilon\left(0 ; c, k c^{\sigma^{-1}}, k^{\sigma} c^{2}+\left(k^{\sigma} c^{2}\right)^{\sigma^{-1}}\right)+\left(0 ; 0,0, c y^{\sigma}+\left(c y^{\sigma}\right)^{\sigma^{-1}}\right) \\
(\varepsilon ; 0, y, 0) L(c, 1)=\varepsilon\left(0 ; k^{-\sigma}(1 / c),(1 / c)^{\sigma^{-1}}, k^{-\sigma}(1 / c)^{2}+\left(k^{-\sigma}(1 / c)^{2}\right)^{\sigma^{-1}}\right) \\
+\left(0 ; k^{-\sigma} y^{\sigma}, y, k^{-\sigma}(1 / c) y^{\sigma}+\left(k^{-\sigma}(1 / c) y^{\sigma}\right)^{\sigma^{-1}}\right),
\end{gathered}
$$

where we understand $1 / c=0$ in the expression of $L(c, 1)$ for $c=0$. (Note that $(0 ; x, 0,0)^{\rho}=\left(0 ; 0, k x^{\sigma^{-1}}, 0\right)$ and $\kappa_{c}=(1 / c)^{\sigma^{-1}}$ for $c \in F$ with $c \neq 0$ and $\kappa_{0}=0$.) Thus applying the permutation $\pi$ on $F \times \mathbb{F}_{2}$ given by $(c, 0)^{\pi}=(c, 0)$, $(0,1)^{\pi}=(0,1)$ and $(c, 1)^{\pi}=\left(k^{-\sigma}(1 / c), 1\right)$, we have

$$
\begin{aligned}
(\varepsilon ; 0, y, 0) L\left((c, \delta)^{\pi}\right)=\varepsilon\left(0 ; c, k c^{\sigma^{-1}}, k^{\sigma} c^{2}+\left(k^{\sigma} c^{2}\right)^{\sigma^{-1}}\right) & \\
& +\delta\left(0 ; k^{-\sigma} y^{\sigma}, y, 0\right)+\left(0 ; 0,0, c y^{\sigma}+\left(c y^{\sigma}\right)^{\sigma^{-1}}\right)
\end{aligned}
$$

for any $\varepsilon \in \mathbb{F}_{2}, y \in F$ and $(c, \delta) \in B_{2} \times \mathbb{F}_{2}$. From this equation, we can easily conclude that

$$
L\left(\left(c_{1}, \delta_{1}\right)^{\pi}\right)+L\left(\left(c_{2}, \delta_{2}\right)^{\pi}\right)=L\left(\left(c_{1}+c_{2}, \delta_{1}+\delta_{2}\right)^{\pi}\right)
$$







\section{References}

[1] U. Dempwolff and Y. Edel, Dimensional dual hyperovals and APN functions with translation groups, J. Algebraic Combin. 39 (2014), 457-496.

[2] A. Pasini and S. Yoshiara, On a new family of flag-transitive semibiplanes, European J. Combin. 22 (2001), 529-545.

[3] _ New distance regular graphs arising from dimensional dual hyperovals, European J. Combin. 22 (2001), 547-560.

[4] H. Taniguchi, Talk at the Kobe Gakuin University on March 3, 2012 and personal communications during March 4-6.

[5] H. Taniguchi and S. Yoshiara, On dimensional dual hyperovals $\mathcal{S}_{\sigma, \phi}^{d+1}$, Innov. Incidence Geom. 1 (2005), 197-219.

[6] _ A new construction of the $d$-dimensional Buratti-Del Fra dual hyperoval, European J. Combin. 33 (2012), 1030-1042.

[7] _ A unified description of four simply connected dimensional dual hyperovals, European J. Combin. 36 (2014), 143-150.

[8] S. Yoshiara, A family of $d$-dimensional dual hyperovals in $P G(2 d+1,2)$, European J. Combin. 20 (1999), 589-603.

[9] __ Dimensional dual arcs—a survey, pp. 247-266, in: Finite Geometries, Groups, and Computation, eds. A. Hulpke, B. Liebler, T. Penttila, and A. Seress, Walter de Gruyter, Berlin-New York, 2006.

Satoshi Yoshiara

Department of Mathematics, TOKYo Woman's Christian University, 2-6-1 ZempukU-Ji, SugINAMI, TOKYO, 167-8585 JAPAN

e-mail: yoshiara@lab.twcu.ac.jp 